\title{
Análise acústica das consoantes líquidas do Português Brasileiro em crianças com e sem transtorno fonológico
}

\section{Acoustical analysis of the liquid consonants of Brazilian Portuguese in children with and without phonological disorder}

\author{
Luciana de Oliveira Pagan ${ }^{1}$, Haydée Fiszbein Wertzner ${ }^{2}$
}

\begin{abstract}
RESUMO
Objetivo: Descrever e comparar os formantes F1, F2 e F3 das líquidas $/ 1, \kappa$, / produzidos por crianças com e sem alterações de fala. Métodos: Cinqüienta e nove crianças foram separadas em três grupos: Grupo Controle (GC), com ausência de alterações no desenvolvimento de fala e linguagem; Grupo Experimental 1 (GE1), apresentando o processo de simplificação de líquidas e/ou de simplificação do encontro consonantal; e Grupo Experimental 2 (GE2), com simplificação de líquidas e outros processos quaisquer. As crianças foram solicitadas a repetir as sílabas la, li, lu; ra, ri, ru e Ka, Ki, Ku e os vocábulos /se'bola/, /'lãma/, /'miKu/, /Zaka're/, /3i' rafa/, /pa' Kasu/ que foram armazenados diretamente no Computerized Speech Laboratory e analisados acústica e estatisticamente. Resultados: O GC apresentou diferenças nítidas entre os três sons estudados em relação aos formantes. Os valores do F3 foram os que mais se modificaram entre os grupos. Os sons produzidos de forma distorcida não apresentaram diferenças significantes entre GE1 e GE2 e nas produções substituídas houve diferença somente na consoante / $/$ / em relação ao F3. Conclusões: As alterações de líquidas encontradas nos sujeitos com transtorno fonológico tiveram características semelhantes, independentemente do fato de terem alterados outros sons da fala, associados ou não.
\end{abstract}

DESCRITORES: Acústica da fala; Transtornos do desenvolvimento de linguagem; Linguagem infantil; Distúrbios da fala; Qualidade da voz; Desenvolvimento infantil

\section{INTRODUÇÃO}

Durante o desenvolvimento da linguagem observa-se, quanto ao aspecto fonológico, um domínio gradativo dos sons da fala tanto no que diz respeito a sua percepção e produção como na compreensão das regras lingüísticas que regem sua utilização numa determinada língua.

$\mathrm{O}$ desenvolvimento da fala é influenciado pelas capacidades perceptivas da criança. Assim, as crianças manifestam a capacidade fisiológica de produzir todos os sons da língua, mas estes sons não são lingüisticamente utilizados até que a

(1) Pós-graduanda em Lingüística e Semiótica Geral pela Faculdade de Filosofia Letras e Ciências Humanas da Universidade de São Paulo - USP São Paulo (SP), Brasil.

(2) Livre Docente do Curso de Fonoaudiologia do Departamento de Fisioterapia, Fonoaudiologia e Terapia Ocupacional da Faculdade de Medicina da Universidade de São Paulo - USP - São Paulo (SP), Brasil.

Trabalho realizado no Laboratório de Investigação Fonoaudiológica em Fonologia da Universidade de São Paulo - USP - São Paulo (SP), Brasil, com auxílio à pesquisa do CNPq.

Endereço para correspondência: Luciana de Oliveira Pagan. R. Dr. Nogueira Martins, 400/52, Saúde, São Paulo - SP, CEP 04143-020. E-mail: lopagan@ig.com.br

Recebido em: 31/10/2006; Aceito em: 28/5/2007 criança incorpore ao seu sistema de linguagem a base perceptiva que é fundamental para sua identificação. Dessa forma, utilizar o delineamento acústico-temporal dos sons produzidos pelos falantes adultos de uma comunidade lingüística parece ser um bom caminho para determinar a base acústica dos erros fonológicos ${ }^{(1-2)}$.

Em geral, a literatura estrangeira e brasileira mostra que os últimos sons a serem adquiridos, nas línguas em que estão presentes, são os líquidos laterais e vibrantes ${ }^{(3-8)}$.

As líquidas do Português Brasileiro são $/ 1 /, /$ / e $/ / /^{(9-10)}$. É interessante mostrar que as líquidas, além de serem bastante afetadas no distúrbio fonológico ${ }^{(9-12)}$, surgem tardiamente no desenvolvimento de crianças e, durante esse período, são muito confundidas com a semivogal $/ \mathrm{w} /$ no inglês e com o /y/ no Português. É possível que esta confusão ocorra porque as crianças não reconhecem uma ou mais características acústicas que os adultos utilizam para distinguir estes sons.

Um estudo ${ }^{(13)}$ sobre a estimulabilidade das consoantes líquidas verificou que o som mais alterado, seja por distorção ou por substituição, foi a vibrante simples / / / seguida das líquidas $/ K /$ e $/ 1 /$, respectivamente. $\mathrm{O} / \mathrm{l} /$ foi a líquida em que menos alterações foram observadas tanto nas crianças com desenvolvimento típico, quanto nas que tinham distúrbio fonológico. 


\section{Características acústicas}

Com o decorrer dos anos, surgiu um grande interesse na obtenção de medidas mais objetivas que pudessem descrever a produção de fala de crianças com desenvolvimento típico e de crianças com distúrbio fonológico.

Para tanto, é importante estudar as propriedades acústicas dos sons, uma vez que muitos autores acreditam que estas características podem fornecer informações relevantes para o maior entendimento sobre o funcionamento do trato vocal, tanto para o diagnóstico como para o tratamento de problemas articulatórios.

A fonética acústica oferece condições de estabelecer uma descrição mais ampla dos sons da fala quando comparada à fonética articulatória justamente por tratar-se de uma classificação geral para todas as línguas.

Uma das formas mais utilizadas atualmente para este fim é a análise acústica que procura avaliar o sinal acústico da fala, ou seja, um evento físico que contém a mensagem lingüística $^{(14-15)}$. A análise acústica espectrográfica procura caracterizar a acústica da onda sonora vocal, ou seja, os sons da fala em termos articulatórios, baseada na postura da língua, e em termos acústicos propriamente ditos, baseada nos formantes e em suas transições ${ }^{(16-17)}$. Este tipo de análise é muito segura e bastante conveniente na avaliação das características de fala de crianças $^{(18-20)}$.

As medidas objetivas, obtidas por meio da análise acústica, possibilitam verificar se a criança apresenta uma inabilidade motora na produção do som ou uma incapacidade em selecionar e utilizar o som de maneira adequada, na produção das palavras. No caso da primeira, esta criança poderia apresentar a omissão de um determinado som, ou produzi-lo de maneira distorcida, enquanto na segunda, o mais comum é que ela substitua o som correto por outro qualquer.

As propriedades acústicas dos sinais de fala explicam a relação existente entre a produção do som pelo falante e sua compreensão pelo ouvinte, uma vez que os mecanismos de percepção captam a pressão das ondas sonoras que constituem a fala. $\mathrm{O}$ falante, para otimizar a comunicação, manipula e explora as características acústicas dos sons da fala possibilitando a transmissão de aspectos prosódicos que facilitam a compreensão pelo ouvinte ${ }^{(21)}$. A inteligibilidade da fala depende crucialmente dos formantes e de sua duração ${ }^{(22)}$.

A espectrografia possui grandes vantagens quando utilizada para complementar as análises fonológicas descritivas por ser de rápida análise, por mostrar precisamente as concentrações de energia na fala e por produzir um espectro que aponta como as concentrações de energia variam no tempo $^{(2,14,23-25)} \mathrm{e}$, por isso, tem sido muito utilizada também como um suplemento à análise perceptiva nos estudos sobre aquisição e desenvolvimento de fala ${ }^{(22)}$.

Um trabalho realizado com consoantes e vogais relata a existência de uma relação geral entre a articulação e os fatores acústicos ${ }^{(26)}$. Tal relação foi dividida em cinco etapas e evidencia como os formantes são influenciados pelas características do trato vocal. Na primeira, a abertura da mandíbula provoca grande crescimento no F1 fazendo com que o F2 cresça se a língua estiver retraída em direção ao palato mole. O F3 pode crescer abruptamente quando houver uma abertura moderada da mandíbula e quando a língua estiver voltada para a região do palato. Na segunda etapa, a movimentação da língua na direção antero-posterior provoca um modesto aumento no F1 (normalmente em torno de $200 \mathrm{~Hz}$ ). $\mathrm{O}$ movimento da frente para uma posição neutra resulta em uma grande queda no F2 em todos os casos. Na terceira etapa, o formato da língua que controla o grau de constrição do trato (assumindo uma posição constante da mandíbula), tem pouco efeito sobre o F1, porém, faz com que os valores de F2 caiam substancialmente enquanto a constrição aumenta. $\mathrm{Na}$ quarta etapa, o arredondamento labial tem o efeito geral de abaixar todas as freqüências de formantes, sendo os efeitos mais fortes observados no F2 e no F3. Na quinta etapa o abaixamento da laringe faz com que o trato vocal se alongue e tenda a abaixar todos os formantes. Normalmente, a movimentação laríngea influencia mais o F2 e o F4 do que o F3.

As consoantes líquidas escolhidas para este estudo tendem a apresentar uma estrutura de formantes muito similar à das vogais, e podem ser produzidas com uma grande variedade articulatória em todas as línguas do mundo ${ }^{(27)}$.

Em estudo $^{(28)}$ realizado com a análise acústica das líquidas $/ 1 /$, / / / e $/ K /$ do Português Brasileiro utilizando a produção de um professor universitário paulistano do sexo masculino de 52 anos de idade, a autora observou que a produção das três líquidas diferiram entre si qualitativa e quantitativamente. Apesar desta diferença, os valores dos três formantes, F1, F2 e F3, mostraram-se muito próximos e, principalmente, os valores do primeiro formante, F1, sugeriram a tendência de proximidade entre essas consoantes.

Outro estudo $^{(29)}$ foi realizado com 23 sujeitos adultos e teve como objetivo descrever as consoantes líquidas do Português Brasileiro em relação à freqüência e à duração. Os resultados indicaram que, em relação à variável frequiência de som, o F1 apresentou médias e desvios padrão para as consoantes $/ \mathrm{l} / \mathrm{e} / \mathrm{K} /$ semelhantes, porém menores para a consoante /r/. No F2, não foram observadas grandes diferenças entre os desvios padrão, enquanto que as médias correspondentes às consoantes $/ K / \mathrm{e} / \mathrm{r} /$ pareceram ser semelhantes e maiores do que as obtidas para a consoante /1/. As médias do F3 diferenciaram os três fonemas sendo o valor mais alto obtido para a consoante $/ K /$, seguida da consoante $/ \mathrm{r} /$ e por último, o menor valor foi o da consoante /1/. Além disso, ao analisar as espectrografias das consoantes /l/ e / $/$.

Existem ainda poucos estudos envolvendo a análise acústica da fala no Brasil, principalmente no que se refere às consoantes líquidas laterais e à vibrante /r/.

Assim, baseado na revisão da literatura, o presente estudo tem como objetivos: descrever e comparar os formantes F1, F2 e F3 das líquidas laterais /l/ e / $/$ / e da líquida vibrante /r/ em três grupos distintos de crianças além de analisar os formantes das líquidas laterais e da vibrante / $\mathrm{f} /$ produzidas corretamente e com algum tipo de alteração.

\section{MÉTODOS}

A presente pesquisa foi aprovada pelo Comitê de Ética em Pesquisa da Universidade de São Paulo sob o número 
481/01. Todos os pais ou responsáveis pelos sujeitos assinaram o Termo de Consentimento Pós-Informação.

Foram sujeitos desta pesquisa 59 crianças, 37 do sexo masculino e 22 do feminino, separados em três grupos denominados de Grupo Controle (GC), Grupo Experimental 1 (GE1) e Grupo Experimental 2 (GE2).

O Grupo Controle (GC) foi constituído por 39 sujeitos, sem queixa de desenvolvimento de fala e linguagem, com idade compreendida entre 6;0 e 9;11 anos. Foram estabelecidos como critérios de inclusão para este grupo, a ausência de alterações nas provas de Fonologia do ABFW Teste de Linguagem Infantil $^{(30)}$, a ausência de queixas de alterações no desenvolvimento de fala e linguagem e a ocorrência de, no máximo, três episódios de otites de repetição.

O Grupo Experimental 1 (GE1) foi composto por $10 \mathrm{su}-$ jeitos com distúrbio fonológico, com idade mínima de 5;0 anos e máxima de 10;2, que deveriam apresentar somente o processo de simplificação de líquidas / $/$ /, / / e / $/ K /$ podendo este vir acompanhado, ou não, do processo de simplificação do encontro consonantal.

O Grupo Experimental 2 (GE2) também foi constituído por 10 sujeitos com distúrbio fonológico, com idade mínima de 5;0 anos e máxima de 12:0, que apresentaram outros processos quaisquer além do processo de simplificação de líquidas ///, /r/ e / / / e de simplificação do encontro consonantal.

O critério de inclusão dos sujeitos no GE1 e no GE2 foi o diagnóstico do Distúrbio Fonológico com a presença do processo fonológico de simplificação de líquidas em três ou mais vocábulos na prova de imitação e na prova de nomeação que compõem a avaliação fonológica. Além disso, essas crianças não poderiam ter realizado qualquer tipo de intervenção fonoaudiológica anteriormente.

Os sujeitos com distúrbio fonológico, dos grupos GE1 e GE2 foram provenientes do Laboratório de Investigação Fonoaudiológica em Fonologia (LIF - Fonologia) do Departamento de Fisioterapia, Fonoaudiologia e Terapia Ocupacional da Faculdade de Medicina da Universidade de São Paulo. As crianças do grupo controle (GC) foram provenientes de uma escola particular da região do Butantã, na cidade de São Paulo.

\section{Material}

A seleção dos sujeitos do Grupo Controle (GC) foi realizada através da aplicação de um questionário específico, elaborado para esta pesquisa, contendo perguntas específicas sobre a aquisição e o desenvolvimento de fala e da audição e das provas de Fonologia do ABFW - Teste de Linguagem Infantil $^{(30)}$. Foi solicitada a assinatura do Termo de Consentimento Pós-Informação.

Nos Grupos Experimentais 1 e 2, foram aplicados todos os testes usados no processo de diagnóstico do LIF Fonologia, entre eles a anamnese, as provas do ABFW Teste de Linguagem Infantil ${ }^{(31)}$ e as provas do Teste de Sensibilidade Fonológica ${ }^{(32)}$. Todas as provas para a seleção dos sujeitos foram gravadas em Compact Disc.

Além disso, foram utilizados, para todos os grupos, os Protocolos de Registro para a Análise Acústica das Conso- antes Líquidas Laterais e Vibrantes ${ }^{(33)}$. Foram criados dois protocolos diferentes para atender às duas listas de repetição realizadas alternadamente entre as crianças.

Para padronizar a apresentação das sílabas com as consoantes líquidas laterais e vibrantes, estas foram previamente gravadas pela própria pesquisadora num Compact Disc da marca EMTEC-BASF (CD-R maxima, digital áudio, de 74 minutos) no gravador de Compact Disc da marca Philips, modelo CDR-785. Utilizou-se também um aparelho de som portátil da marca AKAI (AJ-C010II) para que o Compact Disc pudesse ser reproduzido.

Os dados obtidos através da repetição de sílabas foram coletados e armazenados diretamente no CSL (Computerized Speech Laboratory, fabricado pela Kay Elemetrics - modelo 4300B) com a utilização de um microfone unidirecional da marca Shure, modelo SM-58. Em seguida, as amostras de fala foram analisadas espectrograficamente no próprio CSL.

\section{Procedimento}

Todos os grupos foram avaliados na mesma sala e individualmente pela primeira autora deste artigo. Foram necessárias três sessões com duração de uma hora cada para a avaliação de cada criança dos três grupos.

As sílabas selecionadas para esta pesquisa foram la, li, lu; ra, ri, ru e $\mathrm{Ka}, \widehat{\mathrm{i}}, \mathrm{Ku}$, ordenadas três a três, de modo que fossem repetidas três vezes cada uma, totalizando 27 repetições.

Para a aplicação das listas de sílabas, a examinadora explicou à criança que ela iria ouvir algumas palavras bem pequenininhas e que ela deveria repeti-las. Antes da gravação, foi realizado um treino com três sílabas que, neste momento, foram ditas pela própria pesquisadora, através da seguinte ordem: "Repita la, ra e Ka".

Logo após a explicação e a certificação de que a criança havia realmente entendido a ordem, realizou-se a gravação. Para manter a fidedignidade da gravação no CSL, a taxa de amostragem (sampling rate) foi ajustada para 14000 amostras por segundo.

A partir da gravação da amostra de fala das crianças realizada no CSL, foi feita a transcrição fonética das 27 emissões de cada sujeito no próprio Protocolo de Registro para a Análise Acústica das Consoantes Líquidas Laterais e Vibrantes para que os processos fonológicos pudessem ser analisados e classificados e para verificar qual o tipo de erro apresentado pelos sujeitos do GE1 e GE2. Este Protocolo não foi preenchido para as crianças do GC, pois elas não apresentavam nenhuma alteração de fala e, portanto, não apresentaram erros que pudessem ser transcritos.

A análise espectrográfica foi realizada para todos os sujeitos dos três grupos. Para tanto, cada uma das emissões foi analisada em forma de onda e em espectrografia.

A separação entre a consoante a ser analisada (/1/, /r/ ou $/ K /$ ) e a vogal subseqüente foi realizada de maneira visual e auditiva. Para o presente estudo foram utilizados somente a média e o desvio padrão dos três primeiros formantes (F1, F2 e F3) obtidos através dos dados descritivos fornecidos pelo próprio programa. 
Quadro 1. Valores médios de F1, F2 e F3 na repetição das sílabas com os sons estudados para os três grupos

\begin{tabular}{|c|c|c|c|c|c|c|c|c|c|}
\hline & \multicolumn{3}{|c|}{$\mathrm{GC}$} & \multicolumn{3}{|c|}{ GE1 } & \multicolumn{3}{|c|}{ GE2 } \\
\hline & $\mathrm{F} 1$ & $\mathrm{~F} 2$ & F3 & $\mathrm{F} 1$ & F2 & F3 & $\mathrm{F} 1$ & $\mathrm{~F} 2$ & F3 \\
\hline$/ \mathrm{la} /$ & 454,06 & 1237,9 & 2116,95 & 422,62 & 1123,18 & 2109,38 & 425,07 & 1128,39 & 2187,19 \\
\hline /li/ & 309,80 & 1747,65 & 2855,50 & 311,36 & 1669,30 & 2763,88 & 334,41 & 1400,41 & 2580,33 \\
\hline$/ \mathrm{lu} /$ & 324,86 & 1279,84 & 2348,33 & 326,68 & 1262,05 & 2341,69 & 347,14 & 1309,60 & 2245,94 \\
\hline /ra/ & 408,07 & 1529,23 & 2330,27 & 450,79 & 1299,90 & 2300,83 & 417,13 & 1186,61 & 2248,98 \\
\hline$/ \mathrm{ri} /$ & 288,64 & 1709,59 & 2601,66 & 324,08 & 1580,37 & 2651,13 & 347,47 & 1279,13 & 2607,03 \\
\hline /ru/ & 317,58 & 1495,55 & 2220,80 & 330,86 & 1365,90 & 2242,18 & 346,31 & 1253,20 & 2227,19 \\
\hline$/ \mathrm{Ka} /$ & 354,06 & 1598,71 & 2879,28 & 353,92 & 1266,95 & 2872,95 & 397,79 & 1216,56 & 2528,01 \\
\hline / $/ \mathrm{i} /$ & 306,88 & 1795,33 & 2841,78 & 298,10 & 1526,29 & 2771,63 & 317,18 & 1172,69 & 2566,01 \\
\hline$/ \mathrm{Ku} /$ & 295,36 & 2072,09 & 2708,48 & 303,76 & 1854,45 & 2737,57 & 321,70 & 1564,77 & 2386,27 \\
\hline
\end{tabular}

A técnica estatística utilizada para este trabalho foi a ANOVA - Analisys of Variance e a comparação dois a dois de ANOVA. O nível de significância adotado foi de 0,05 .

\section{RESULTADOS}

\section{Descrição dos valores de F1, F2 e F3 para /l/, /f/ e /K/}

Para essa análise foram considerados os valores médios dos formantes F1, F2 e F3 na produção dos sons /1/, / / e / / / nas três repetições solicitadas a cada criança, em sílabas, independentemente das vogais subseqüentes. No Quadro 1 são apresentados os valores médios das três repetições reunidas de F1, F2 e F3 para as sílabas analisadas para cada um dos três grupos.

A média dos valores foi realizada considerando-se todas as produções do GC e somente as produções corretas e/ou distorcidas do GE1 e do GE2 (foram excluídas do cálculo as sílabas e palavras cujo som alvo foi omitido).

Nas Figuras 1, 2 e 3 observa-se a análise espectrográfica obtida a partir da emissão da sílaba /la/ por um sujeito do GC, do GE1 e do GE2, respectivamente. Entre as barras verde e vermelha está a sílaba selecionada e entre as barras verde e azul está o som alvo /1/.

A ANOVA revelou diferenças estatisticamente significantes entre os três grupos na produção do som /1/ em relação aos valores de F1 (p=0,001) e F2 ( $\mathrm{p}=0,029)$, mas não para F3 $(\mathrm{p}=0,455)$. Entretanto, quando analisadas qualitativamente as espectrografias, esta diferença não pode ser ob-

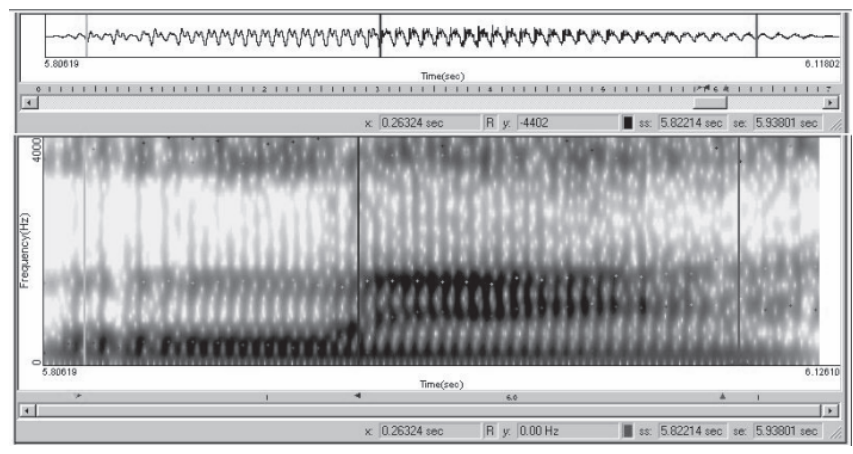

Figura 1. Forma de onda e espectrograma da emissão da sílaba /la/ por um sujeito do GC

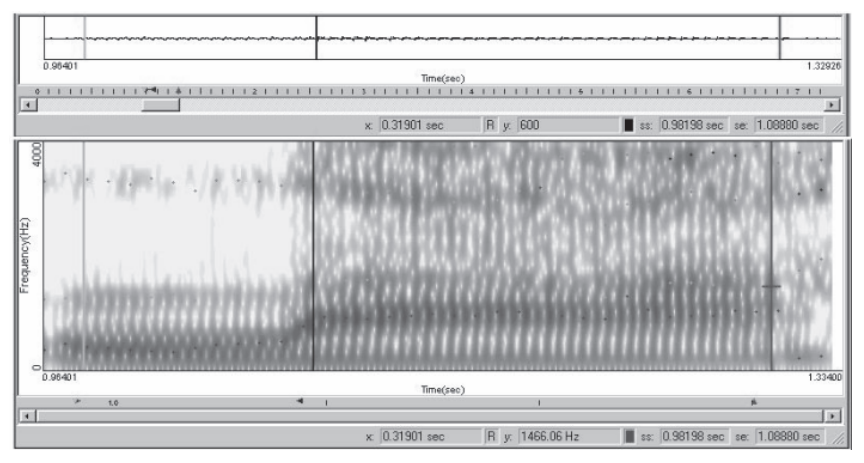

Figura 2. Forma de onda e espectrograma da emissão da sílaba /la/ por um sujeito do GE1

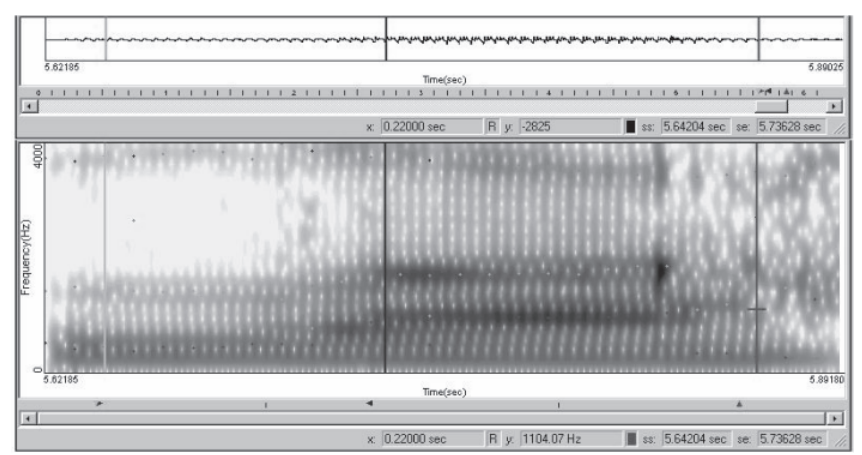

Figura 3. Forma de onda e espectrograma da emissão da sílaba /la/ por um sujeito do GE2

servada em relação à forma de onda e à duração do som, que é bastante semelhante para os representantes dos três grupos. Isto demonstra que o som /1/ é percebido auditivamente de modo semelhante pelos três grupos, mas que produzem uma diferenciação acústica importante, principalmente nos duas primeiras freqüências formantes.

Nas Figuras 4, 5 e 6 observa-se a análise espectrográfica obtida a partir da emissão da sílaba /ra/ por um sujeito do GC, do GE1 e do GE2, respectivamente. O sujeito do GC produziu a sílaba corretamente, o do GE1 e do GE2 produziram a mesma sílaba de maneira distorcida. Entre as barras verde e vermelha está a sílaba selecionada e entre as barras verde e azul está o som alvo /r/, da maneira como foi produzido.

Nesta análise, as freqüências formantes F1 $(p=0,169)$ e F3 ( $p=0,136)$ não apresentaram significância estatística que 
só foi observada para F2 ( $\mathrm{p}<0,001)$. A forma de onda e o espectrograma obtidos a partir da emissão de uma criança do GC permitem verificar que o /s/ é um som de curta duração, com formantes bem definidos. Na emissão dos outros dois grupos, GE1 e GE2, observa-se que o mesmo som foi produzido de maneira distorcida, já que apresenta tempo de duração mais longo e formantes espalhados ao longo do espectrograma, assemelhando-se muito às características espectrográficas apresentadas na produção do som /1/

Nas Figuras 7, 8 e 9 observa-se a análise espectrográfica obtida a partir da emissão da sílaba $/ \Lambda \mathrm{a} /$ realizada por um sujeito do GC, GE1 e GE2, respectivamente. O sujeito do GE1 produziu este som de maneira distorcida, enquanto o do GE2 produziu-o como um /ia/. Entre as barras verde e vermelha está a sílaba selecionada, e entre as barras verde e

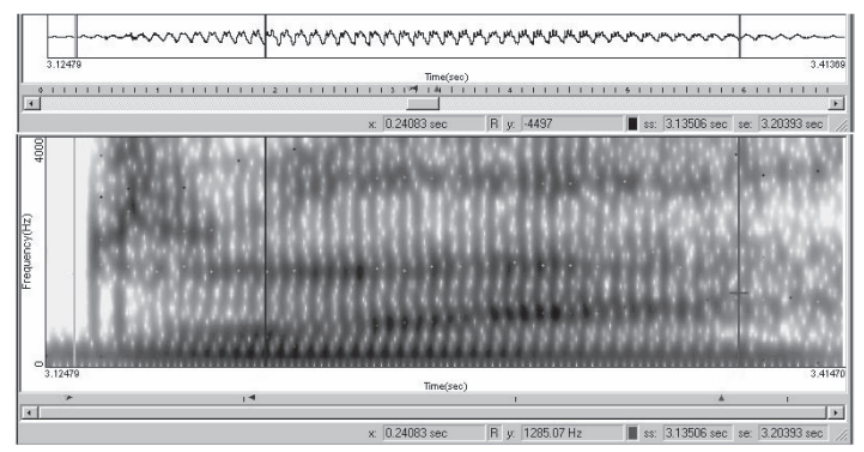

Figura 4. Forma de onda e espectrograma da emissão da sílaba/ra/ por um sujeito do GC

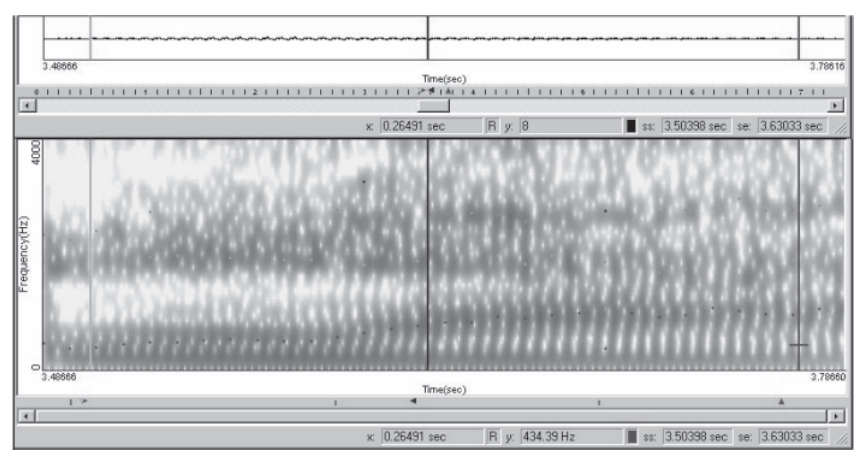

Figura 5. Forma de onda e espectrograma da emissão da sílaba /ra/ por um sujeito do GE1

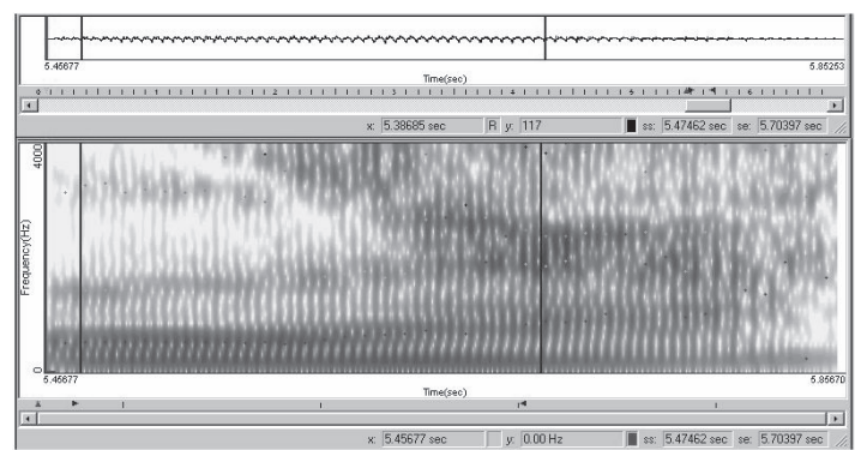

Figura 6. Forma de onda e espectrograma da emissão da sílaba/ra/ por um sujeito do GE2 azul está o som alvo $/ K /$, da maneira como foi produzido.

Para este som, foi observada significância estatística para os três primeiros formantes F1, F2 e F3 ( $p<0,001)$. No caso dessas análises, os dados encontrados permitem verificar que tanto a forma de onda quanto o espectrograma do som $/ K /$ assemelham-se às características apresentadas para o som /1/. No GE1 e, no GE2, como ele foi substituído pela vogal /i/, pode-se notar características espectrográficas desta vogal.

Além disso, a concentração de energia obtida na emissão deste som pelos três grupos está deslocada para a região superior do gráfico, o que demonstra que, de certa maneira, as crianças tentaram aproximar sua produção àquela apresentada pelo GC levando a crer que tenham o conhecimento sobre as características do som, porém, uma dificuldade motora as impossibilitou de produzi-lo de maneira correta.

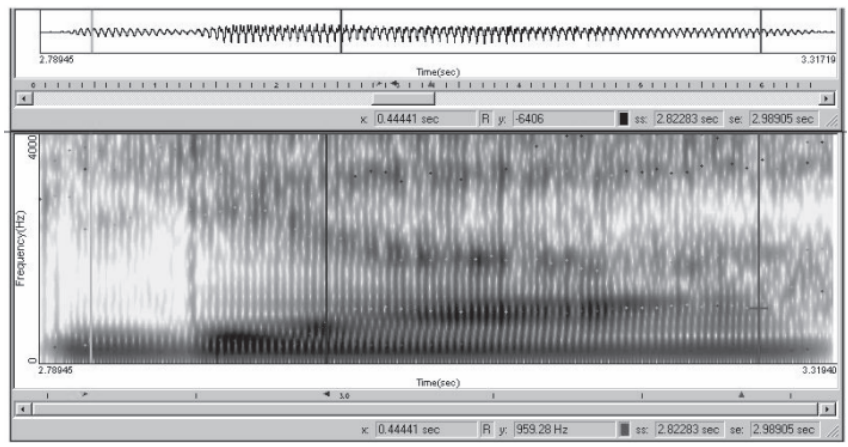

Figura 7. Forma de onda e espectrograma da emissão da sílaba / $\mathrm{Ka/}$ por um sujeito do GC

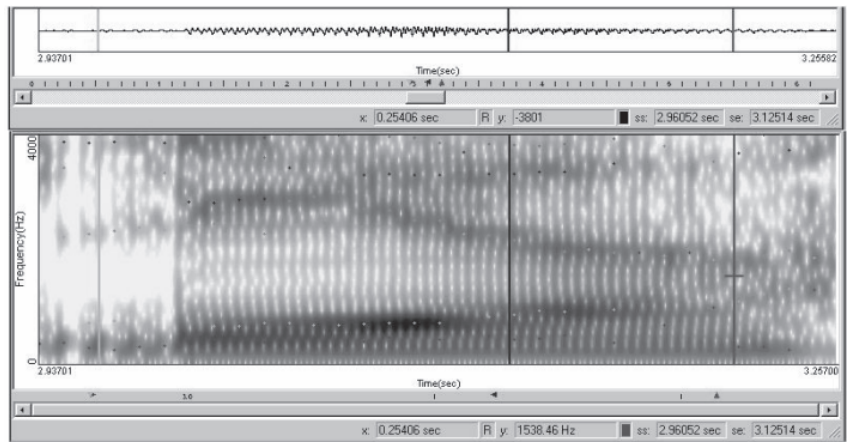

Figura 8. Forma de onda e espectrograma da emissão da sílaba / Ka/ por um sujeito do GE1

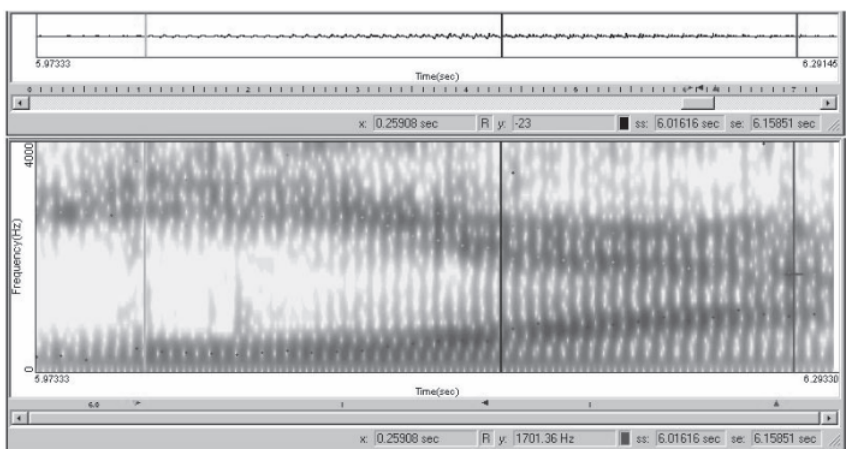

Figura 9. Forma de onda e espectrograma da emissão da sílaba / $/ \mathrm{Ka} /$ por um sujeito do GE2 


\section{DISCUSSÃO}

As crianças dos grupos GE1 e GE2, com idades compreendidas entre 5;0 e 12;0 anos, ainda não haviam adquirido, quando analisadas para esta pesquisa, um ou mais dos dois sons líquidos laterais e/ou da vibrante /s/ do Português Brasileiro. Isto mostra que, embora estas crianças já estivessem na idade de possuir estes sons internalizados em seu sistema fonológico, elas não foram capazes de adquiri-los de maneira adequada, o que é esperado e observado nas crianças da mesma idade sem alteração de fala.

$\mathrm{Na}$ presente pesquisa, a análise acústica contribuiu para diferenciar a produção dos três sons /l/, / / e /K/ em diversos contextos articulatórios, tanto no GC como no GE1 e no GE2. Entretanto, no GC, foram observadas diferenças mais evidentes entre os três sons estudados, em relação aos formantes. Também foi possível verificar que as crianças do GE1 comportaram-se de modo diferente das do GE2 em algumas situações.

Em relação aos valores dos formantes, apesar de F1, F2 e F3 terem apresentado diferenças entre si, os valores de F2 foram os que mais se diferenciaram para os três sons, tanto no estudo intragrupo como no estudo entre os grupos. $\mathrm{Na}$ comparação dos valores dos formantes entre os grupos (GC X GE1 X GE2), pode-se notar que os valores do F2, de maneira geral, foram os que mais se modificaram de um grupo para outro nas três líquidas, o que indica ser este formante importante na diferenciação dos sons líquidos laterais e vibrantes.

Especificamente para o som $/ K /$, na comparação entre $/ 1 /$, /f/ e $/ K /$, o F3 mostrou valores bastante diferenciados e estatisticamente significantes, quando comparados aos obtidos na produção do $/ 1 /$ e do $/ \mathrm{f} /$, mostrando ser este formante o grande responsável pelo reconhecimento da líquida palatal nos três grupos.

Estes achados corroboram os resultados dos estudos realizados para a língua inglesa ${ }^{(1,2,15,26)}$. Estes autores afirmam que o F1 não serve para diferenciar as líquidas por apresentar valores de baixa frequiência muito próximos tanto para o /l/ quanto para o /r/. No Brasil, outro estudo reafirmou estes $\operatorname{achados}^{(28)}$ e acrescentou ainda que o F3 é capaz de diferenciar a líquida lateral das líquidas alveolar e vibrante, por possuir valores mais altos.

Comparados aos valores obtidos no estudo anterior ${ }^{(29)}$ para o Português Brasileiro, os resultados obtidos nesta pesquisa indicaram valores menores de F1 para o som /r/. Entretanto, os achados em relação ao F2 e F3 foram semelhantes aos deste estudo, no qual os valores foram menores para as sílabas com o som /1/, independentemente do grupo em que foram analisados. De modo geral, os resultados obtidos em relação ao valor médio de F1 e F2 para os sons /1/ e /s/ coincidem com outro estudo ${ }^{(34)}$ que apresentou os valores de F1 por volta de 250 a $500 \mathrm{~Hz}$ e os de F2, entre 1250 e $1460 \mathrm{~Hz}$ para falantes do inglês.

Independentemente da vogal subseqüente, observou-se diferenças significantes (ANOVA) entre os três formantes obtidos na produção de sílabas somente no GC, o que indica que estas crianças realmente produzem de modo diferencia- do os sons analisados. O mesmo resultado não foi observado nos grupos GE1 e GE2 demonstrando que a produção destas crianças é realizada de modo indiferenciado, ou seja, não há, em sua produção, a nítida distinção entre $/ 1 / \neq \mid \kappa / \neq /$ r $/$ para F1, F2 e F3 como foi observado no GC.

As diferenças entre os formantes, que puderam ser observadas tanto no GE1 quanto no GE2, foram muito pequenas, o que comprova que, apesar do GE1 possuir apenas o processo fonológico de simplificação de líquidas e/ou do encontro consonantal e o GE2 possuir, além desses, outros processos, em relação às consoantes líquidas, ambos se comportam de maneira semelhante. Esta não diferenciação presente na comparação entre os formantes foi mais evidente para os grupos GE1 e GE2 na produção dos sons $/ \mathrm{s} /$ e $/ K /$, sendo o /l/ melhor diferenciado. Isso indica que os sons $/ \mathrm{r} / \mathrm{e}$ $\mathrm{I} / \mathrm{K}$ foram produzidos de modo muito semelhante, quer seja por substituição ou por distorção.

Durante a análise espectrográfica dos sons que foram produzidos de forma distorcida, não foram observadas diferenças significantes entre os dois grupos experimentais, apesar do GE1 tê-las apresentado em menor quantidade. Este fato comprova, mais uma vez, que a maneira como os sujeitos dos dois grupos produzem as três líquidas estudadas independe da quantidade de processos fonológicos que possam estar associados ao de simplificação de líquidas e/ou do encontro consonantal. Além disso, as distorções apresentadas pelo GE1 e pelo GE2 são bastante parecidas, o que indica alguma conformação semelhante do trato vocal no momento da produção das sílabas e palavras com este som.

Já para as substituições, a única diferença observada entre o GE1 e GE2 foi na consoante $/ K /$ em relação ao F3. Isto retoma o fato do F3 ser realmente um grande diferenciador deste fonema em relação às outras duas líquidas. Mesmo sendo substituído por outros sons, suas características articulatórias parecem se manter, fazendo com que o F3 continue a diferenciá-lo das demais.

Esses resultados demonstram a importância da obtenção de dados precisos para o diagnóstico e a posterior intervenção fonoaudiológica. A verificação da forma como a criança está produzindo um determinado som por meio da análise espectrográfica auxilia na adequação de sua produção, além de fornecer um parâmetro de comparação dos dados de fala.

Assim, por meio da realização deste trabalho pretende-se contribuir para o diagnóstico de crianças que apresentem, em sua fala, os processos fonológicos de simplificação de líquidas ou de simplificação do encontro consonantal facilitando assim, a intervenção terapêutica que deverá ser seguida. Outros estudos devem ser realizados sobre este assunto já que a análise espectrográfica permite muitas outras análises, que muito podem contribuir para a ação do fonoaudiólogo.

\section{CONCLUSÃO}

O estudo realizado mostrou que as alterações de líquidas encontradas nos sujeitos com transtorno fonológico, no que diz respeito às análises espectrográficas, têm características semelhantes, independentemente do fato de terem ou não alterações em outros sons da fala associadas. Acusticamen- 
te, os grupos GE1 e GE2 comportam-se de maneira semelhante com relação às distorções.

Ressalta-se que através da espectrografia foi possível verificar diferenças entre os sons $/ \Lambda /$ e $/ \mathrm{r} /$ produzidos pelo Grupo Controle e pelos dois grupos com transtorno fonológico. Já para o som /1/, que é menos alterado, não foram observa- das diferenças.

Outros estudos envolvendo a análise de líquidas devem ser realizados, principalmente pelo fato de serem estes os sons de maior alteração e dificuldade de produção do Português Brasileiro.

\begin{abstract}
Purpose: To describe and compare the F1, F2 and F3 formants of the liquids $/ 1, \kappa$, s/ produced by children with and without phonological impairment. Methods: Fifty nine children were separated into three groups: Control Group (CG), with absence of disorders in speech and language development; Experimental Group 1 (EG1), with presence of the process of liquids simplification and/or cluster simplification; and Experimental Group 2 (EG2), with liquid simplification and any other processes. Children were requested to repeat the syllables la, li, lu; ra, ri, ru and $\mathrm{Ka}, \mathrm{Ki}, \mathrm{Ku}$ and the words /se'bola/, /'lãma/, /'miKu/, /Zaka'rE/, /zi'rafa/, /pa' Kasu/. Data were stored and analyzed in the Computerized Speech Laboratory. Statistical analyses were provided. Results: Regarding the formants, the CG showed great differences between the three studied sounds. Between-groups analysis showed that F3 presented the greatest modifications. Distorted sounds did not present significant differences between EG1 and EG2 and the substituted ones showed differences in F3 only for the consonant $/ K /$. Conclusions: The liquids substitutions and distortions found in EG1 and EG2 children had similar characteristics, independently of having only the process of liquid simplification or others in their speech.
\end{abstract}

KEYWORDS: Speech acoustics; Language development disorders; Child language; Speech disorders; Voice quality; Child development

\title{
REFERÊNCIAS
}

1. Dalston RM. Acoustic characteristics of English /w, r, 1/ spoken correctly by young children and adults. J Acoust Soc Am. 1975;57(2):462-7.

2. Chaney C. Acoustic analysis of correct and misarticulated semivowels. J Speech Hear Res. 1988;31(2):275-87.

3. Hodson BW, Paden EP. Phonological processes which characterize unintelligible and intelligible speech in early childhood. J Speech Hear Disord. 1981;46(4):369-73.

4. Khan LM. A review of 16 major phonological processes. Lang Speech Hear Serv Sch. 1982;13(2):77-85.

5. Yavas MS. Padrões na aquisição da fonologia do português. Letras Hoje. 1988;23(2):7-30.

6. Wertzner HF. Aquisição da articulação: um estudo em crianças dos três aos sete anos. Estud Psicol (Campinas). 1994;11(1-2):11-21.

7. Goldstein BA, Iglesias A. Phonological patterns in normally developing Spanish-speaking 3- and 4-year-olds of Puerto Rican descent. Lang Speech Hear Serv Sch. 1996;27(1):82-90.

8. Ramos APF. Aquisição dos róticos em um dialeto do português brasileiro: discussão de aspectos fonéticos e fonológicos e suas implicações para a clínica fonoaudiológica. Rev Soc Bras Fonoaudiol. 2000;4(6):31-5.

9. Hernandorena CLM, Lamprecht RR. A aquisição das consoantes líquidas do português. Letras Hoje. 1997;32(4):7-22.

10. Mota HB. Uma abordagem terapêutica baseada nos processos fonológicos no tratamento de crianças com desvios fonológicos. Letras Hoje. 1993;28(10):89-97.

11. Gurgueira AL. Estudo acústico dos fonemas surdos e sonoros do português do Brasil, em crianças com distúrbio fonológico apresentando o processo fonológico de ensurdecimento [tese]. São Paulo: Faculdade de Filosofia, Letras e Ciências Humanas da Universidade de São Paulo; 2000.

12. Wertzner HF, Herrero SF, Iderilha PN, Pires SCF. Classificação do distúrbio fonológico por meio de duas medidas de análise: porcentagem de consoantes corretas (PCC) e índice de ocorrência dos processos fonológicos (PDI). Pró-Fono. 2001;13(1):90-7.

13. Castro MM. Estudo da estimulabilidade de crianças com desenvolvimento típico e com distúrbio fonológico para os fonemas líquidos laterais e vibrante simples [tese]. São Paulo: Faculdade de Filosofia, Letras e Ciências Humanas da Universidade de São Paulo; 2004.

14. Kent RD, Read C. The acoustic analysis of speech. San Diego, Calif.: Singular Pub. Group; c1992.

15. Fowler CA. Listeners do hear sounds, not tongues. J Acoust Soc Am. 1996;99(3):1730-41.

16. Eguchi S, Hirsh IJ. Development of speech sounds in children. Acta Otolaryngol Suppl. 1969;257:1-51.

17. Behlau M, organizadora. Voz: o livro do especialista. Rio de Janeiro: Revinter; c2001. v. 1.

18. Kent RD. Anatomical and neuromuscular maturation of the speech mechanism: evidence from acoustic studies. J Speech Hear Res. 1976;19(3):421-47.

19. Recasens D, Fontdevila J, Pallarés MD. Velarization degree and coarticulatory resistance for /1/ in Catalan and German. J Phonet. 1995;23:37-52.

20. Mezzomo CL. A análise acústica como subsídio para a descrição da aquisição do constituinte coda. Letras Hoje. 2003;38(2):75-82.

21. O'Shaughnessy D. Critique: speech perception: acoustic or articulatory? J Acoust Soc Am.1996;99(3):1726-9.

22. Mattingly IG. Speech synthesis for phonetic and phonological models [text on the Internet]. [cited 2003 Sep 30]. Available from: http:// www.mindspring.com/ ssshp/ssshp_cd/im_home.htm

23. Weismer G, Dinnsen D, Elbert M. A study of the voicing distinction associated with omitted, work-final stops. J Speech Hear Disord. 1981;46(3):320-8.

24. Smit AB, Bernthal JE. Voicing contrasts and their phonological implications in the speech of articulation-disordered children. J Speech Hear Res. 1983;26(4):486-500. 
25. Gierut JA, Dinnsen DA. On word-initial voicing: converging sources of evidence in phonologically disordered speech. Lang Speech. 1986;29(Pt 2):97-114.

26. Lindbloom BE, Sundberg JE. Acoustical consequences of lip, tongue, jaw, and larynx movement. J Acoust Soc Am. 1996;50(4):1166-79.

27. Narayanan S, Byrd D, Kaun A. Geometry, kinematics, and acoustics of Tamil liquid consonants. J Acoust Soc Am. 1999;106(4 Pt 1):19932007.

28. Silva AHP. Para a descrição fonético-acústica das líquidas no português brasileiro: dados de um informante paulistano [tese]. Campinas: Universidade Estadual de Campinas; 1996.

29. Teruya NM, Wertzner HF. Análise acústica das líquidas do português brasileiro. São Paulo; 2000. [Relatório de Iniciação Científica-FAPESP]

30. Wertzner HF. Fonologia. In: Andrade CRF, Befi-Lopes DM, Fernandes FDM, Wertzner HF. ABFW: teste de linguagem infantil nas áreas de fonologia, vocabulário, fluência e pragmática. Carapicuíba: Pró-Fono; 2000. p. 5-40.
31. Andrade CRF, Befi-Lopes DM, Fernandes FDM, Wertzner HF. ABFW: teste de linguagem infantil nas áreas de fonologia, vocabulário, fluência e pragmática. Carapicuíba: Pró-Fono; 2000.

32. Herrero SF. Perfil das crianças: pré-escolares e escolares no teste de sensibilidade fonológica [tese]. São Paulo: Faculdade de Filosofia, Letras e Ciências Humanas da Universidade de São Paulo; 2001.

33. Pagan LO. Estudo das líquidas laterais e vibrantes em crianças com distúrbio fonológico: analise acústica e articulatória [tese]. São Paulo: Faculdade de Filosofia, Letras e Ciências Humanas da Universidade de São Paulo; 2003.

34. Narayanan SS, Alwan AA, Haker K. Toward articulatory-acoustic models for liquid approximants based on MRI and EPG data. Part I. The laterals. J Acoust Soc Am. 1997;101(2):1064-77. 